\title{
ESTRATÉGIAS DE CONTROLE POR MODELO DE REFERÊNCIA DE SISTEMAS A EVENTOS DISCRETOS MAX-PLUS LINEARES
}

\author{
Carlos Andrey Maia* \\ maialcpdee. ufmg.br \\ Rafael Santos Mendes ${ }^{\ddagger}$ \\ rafaeledca.fee.unicamp.br
}

\author{
Ricardo Lüders ${ }^{\dagger}$ \\ luders@dainf.cefetpr.br \\ Laurent Hardouin ${ }^{\S}$ \\ laurent. hardouindistia.univ-angers.fr
}

\author{
*Depto. de Engenharia Elétrica - UFMG \\ Av. Antônio Carlos 6627 - Pampulha \\ 31270-010 Belo Horizonte - MG - Brasil \\ ${ }^{\dagger}$ Centro Federal de Educação Tecnológica do Paraná - CEFET/PR \\ Av. Sete de Setembro, 3165 \\ 80230-901 Curitiba - PR - Brasil \\ ${ }^{\ddagger}$ Faculdade de Engenharia Elétrica e de Computação - UNICAMP \\ DCA/FEEC/UNICAMP-C.P. 6101 \\ 13083-970 Campinas - SP - Brasil \\ ${ }^{\S}$ Laboratoire d'Ingénierie des Systèmes Automatisés - ISTIA/Université d'Angers \\ Av. Notre Dame du Lac 62 \\ 49000 Angers - França
}

\begin{abstract}
This paper deals with the control of discrete event systems modelled by the Max-plus algebra. This approach is used to describe processes whose dynamics are characterized by time delay and synchronization phenomena. Typical examples of these processes are assembly lines in manufacturing systems. The studied control problem is related to the justin-time strategy for production planning. In this tutorial paper, several strategies based on the model reference control approach are presented, exemplified and discussed.
\end{abstract}

KEYWORDS: Discrete Event Systems, System Control, Justin-Time, Max-plus Algebra, Timed Event Graphs.

Artigo submetido em 09/11/2004

1a. Revisão em 06/06/2005;

2a. Revisão em 16/09/2005;

Aceito sob recomendação do Ed. Assoc. Prof. Paulo Eigi Miyagi

\section{RESUMO}

Este artigo trata do controle de sistemas a eventos discretos modelados pela álgebra Max-plus. Esta abordagem é usada para descrever processos cuja dinâmica seja caracterizada por retardos no tempo e fenômenos de sincronização. Exemplos típicos desses processos são as linhas de montagem em sistemas de manufatura. O problema de controle estudado está relacionado à estratégia "just-in-time" em planejamento da produção. Neste artigo tutorial, diversas estratégias baseadas na abordagem por modelo de referência são apresentadas, exemplificadas e discutidas.

PALAVRAS-CHAVE: Sistemas a eventos discretos, Controle de sistemas, Just-in-time, Álgebra Max-plus, Grafos de eventos temporizados. 


\section{INTRODUÇÃO}

De modo geral, um sistema a eventos discretos (SED) é um sistema dinâmico cujas variações de estado são estritamente condicionadas pela ocorrência de eventos. O conceito de evento é fundamental, caracterizado como algo sem duração e única causa possível para as mudanças de estado. Portanto, num SED, as mudanças de estado ocorrem estritamente num conjunto enumerável de instantes de tempo. Diz-se também que um SED tem sua dinâmica dirigida pela ocorrência de eventos. Outro aspecto importante a respeito dos SED é o fato de seu espaço de estados ser normalmente discreto (e em muitas aplicações finito). Essas características distinguem os SED dos sistemas que têm sua dinâmica dirigida pelo tempo e seu espaço de estado contínuo, cuja modelagem é tradicionalmente feita através das equações diferenciais e das equações a diferenças. Esses sistemas serão chamados de sistemas contínuos (mesmo no caso em que o tempo for discreto).

Em um SED os eventos podem ser de vários tipos e programados de maneiras diversas. Podem ocorrer autonomamente ou programados pela ocorrência prévia de algum outro evento e a definição do instante de ocorrência pode ser determinística ou não-determinística (inclusive aleatória). Exemplos típicos de SED são os sistemas de manufatura, o "hardware" de um computador, e em outro nível de abstração, as redes de comunicação. Dois aspectos são de grande importância no estudo da dinâmica de SED: os problemas de conflito e os problemas de sincronização. Os sistemas de manufatura oferecem exemplos desses dois aspectos. Um conflito ocorre num sistema de manufatura quando, por exemplo, duas peças devem ser processadas na mesma máquina, devendo haver uma decisão sobre qual delas será processada em primeiro lugar. $\mathrm{O}$ aspecto de sincronização fica evidente, por exemplo, quando a montagem de uma peça depende de duas ou mais partes, cujo processamento por sua vez pode obedecer às mesmas restrições. Nesse caso, obviamente a parte com processamento mais tardio determinará o início da montagem da peça final. Neste artigo a atenção será concentrada nos sistemas sem conflito, onde predominam os problemas de sincronização.

Até pouco mais de duas décadas atrás, os SED eram tratados predominantemente através de técnicas de simulação (Banks et al., 2000), ou de otimização estática, sendo que os resultados analíticos provinham da Teoria de Filas (Kleinrock, 1975) e da Teoria de Redes de Petri (Murata, 1989). Em particular, esta última abordagem teve grande desenvolvimento durante as décadas de 60 e 70, até o início dos anos 80. A partir desse momento, certamente impulsionadas pelos desafios e exigências do mundo da automação industrial, diversas novas abordagens para o problema de análise e síntese de controladores para SED foram iniciadas, envolvendo as áreas de Teoria de Sistemas, Pesquisa Operacional e Teoria da Computação. São exemplos dessas abordagens entre outras, a Teoria de Controle Supervisório (Ramadge e Wonham, 1989), a Análise de Perturbações (Cassandras e Lafortune, 1999), as técnicas baseadas em Lógica Temporal (Ostroff, 1989) e aquelas baseadas na Álgebra Maxplus (Baccelli et al., 1992). Além disso, significativos desenvolvimentos ocorreram na já existente Teoria de Redes de Petri e na Teoria de Sistemas Híbridos (Antsaklis, 2000), isto é, sistemas que combinam as características de sistemas contínuos e a eventos discretos.

Este artigo é baseado na metodologia Max-plus. Essa abordagem utiliza basicamente uma estrutura algébrica onde a adição é definida como sendo a operação de maximização e a multiplicação como sendo a operação de soma usual. Essa estrutura algébrica vem já sendo estudada há algumas décadas (Cuninghame-Green, 1979). Entretanto, sua utilização na formalização de uma teoria de SED sujeitos à sincronização de eventos é apresentada de maneira significativa por Cohen et al. (1989), sendo posteriormente revisitada (Cohen et al., 1999). A partir daí, diversos trabalhos têm sido publicados sobre a análise e o controle desse tipo de SED.

Particularmente no que se refere ao controle desses sistemas, Cohen et al. (1989) propõem um problema de controle no qual se deseja obter o "melhor" controle possível de maneira a garantir que a saída do sistema siga uma determinada trajetória de referência. A solução desse problema foi genericamente denominada de controle ótimo, no sentido de que o "melhor" controle possível é aquele que atrasa ao máximo o disparo dos eventos de entrada do sistema. Boimond e Ferrier (1996) implementam o controle ótimo, utilizando uma estrutura de controle do tipo IMC ("Internal Model Control"). Já Cottenceau et al. (2001) apresentam um problema de controle no qual se deseja obter um controlador que altere o comportamento entrada-saída do sistema segundo um modelo pré-determinado, denominado modelo de referência. Esse problema é tratado também neste artigo, que reúne e relaciona os resultados obtidos em diferentes trabalhos anteriores (Lüders, 2001; Lüders e Santos-Mendes, 2002; Maia et al., 2003; Maia, 2003). Além disso, propostas de controle adaptativo são formuladas por Menguy et al. (2000). Atualmente, sistemas variantes no tempo têm sido considerados (Lahaye et al., 2004), assim como o projeto de controladores robustos usando matemática intervalar (Lhommeau et al., 2004), ambos na abordagem Max-plus.

Mais formalmente, a metodologia Max-plus fundamenta-se em resultados algébricos relativos aos semi-anéis idempotentes (também chamados de dióides) e na Teoria de Residuação, cujas bases são resumidas na próxima seção. Sua principal característica é descrever a dinâmica de um SED a partir de um sistema de equações algébricas lineares, es- 
critas numa álgebra não convencional. Conforme será visto ainda na Seção 2, esse equacionamento permite a análise de alguns aspectos do desempenho do sistema e, em combinação com a Teoria de Residuação, a síntese de controladores de modo a satisfazer a determinados critérios de desempenho. As redes de Petri também são sumariamente introduzidas na seção 2, tendo como objetivo proporcionar uma ferramenta de modelagem prévia para os SED. Desse modo, ao longo deste artigo, considera-se que um SED é conhecido a partir de uma Rede de Petri que o representa. É importante destacar que a abordagem Max-plus tem como limitação o fato de que seus principais resultados de análise e síntese se aplicam a sistemas sem características de conflito, isto é, a sistemas cuja dinâmica é exclusivamente resultante de suas características de sincronização. Esse fato restringe as redes de Petri que modelam os SED abordados neste trabalho aos Grafos de Eventos Temporizados, também apresentados na seção 2. Para sistemas com esse tipo de restrição, o problema de controle abordado está relacionado com a estratégia "just-in-time" de planejamento da produção, conforme descrito na seção 3. Tomando-se novamente os sistemas de manufatura como exemplo, isto significa que se considera uma dada trajetória ("duedates") como sendo uma referência para o sistema e o objetivo do controlador é retardar ao máximo a entrada de matérias-primas no processo de modo a evitar a formação de estoques no interior da planta, mas atendendo à demanda pré-especificada pela taxa de produção de referência. Finalmente, na seção 4 encontram-se as principais contribuições deste trabalho, sendo apresentadas algumas metodologias específicas para a síntese de controladores baseadas na Teoria de residuação.

\section{PRELIMINARES}

\subsection{Sistemas Max-plus lineares}

Nesta seção, serão introduzidos alguns conceitos relativos aos sistemas a eventos discretos, em particular àqueles para os quais o problema de sincronização é central.

Uma ferramenta clássica para a modelagem de SED são as redes de Petri, cujos resultados principais são propostos por Murata (1989). A discussão a seguir resume o material sobre esse tema, necessário à compreensão do presente artigo. As redes de Petri são definidas como grafos bi-partidos, isto é, com dois tipos de nós, a que chamaremos de lugares e transições. Os arcos do grafo são direcionados e interligam lugares a transições e transições a lugares (mas nunca dois nós do mesmo tipo). Para cada arco direcionado da rede, o nó origem é denominado nó de entrada do nó destino e o nó destino é denominado nó de saída do nó origem. Cada transição da rede é associada a um tipo de evento do SED e os lugares da rede definem as condições sob as quais as suas transições de saída são disparadas. O disparo de uma transição é associado à ocorrência de um evento no SED e é esse conceito que permite modelar a evolução dinâmica do SED. As condições de disparo de uma transição e suas consequiências para a rede são formalizadas através do conceito de marcação da rede. Uma marcação é um mapeamento que associa a cada lugar da rede um número inteiro positivo. É usual dizer que um lugar "contém um número de fichas" igual à sua marcação. Diz-se que uma transição está habilitada, quando todos os lugares de entrada dessa transição contém pelo menos uma ficha. Uma transição só dispara quando está habilitada e após seu disparo cada um dos seus lugares de entrada têm sua marcação decrementada de uma unidade e cada um dos seus lugares de saída têm sua marcação incrementada de uma unidade.

A Figura 1 ilustra a dinâmica de um rede simples. Convém observar que o número de fichas numa rede de Petri não necessariamente se conserva. Pode-se temporizar uma rede de Petri de diversas maneiras, sendo usual fazê-lo atribuindo um atraso a cada lugar (rede p-temporizada) - ver Wang (1998). $\mathrm{O}$ atraso de um lugar é um número inteiro positivo que tem como significado o intervalo de tempo entre o instante em que uma ficha (marcação) é atribuída àquele lugar e o instante em que esta ficha contribui para a habilitação das transições de saída do lugar.

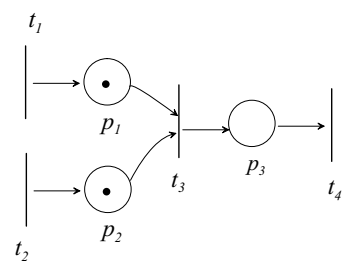

(a)

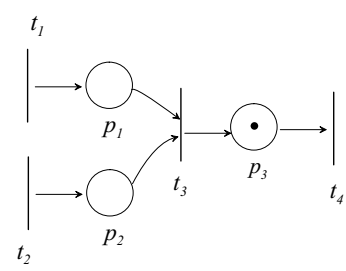

(b)
Figura 1: A Figura (a) mostra a marcação da rede com transição $t_{3}$ habilitada. Em (b) é mostrada a marcação da rede após o disparo de $t_{3}$.

Um Grafo de Eventos Temporizado (GET) é uma Rede de Petri temporizada particular, restrita aos casos em que cada lugar da rede tem uma única transição de entrada e uma única transição de saída. É importante destacar que as Redes de Petri capazes de modelar conflitos num SED necessariamente possuem lugares com mais de uma transição de entrada e/ou mais de uma transição de saída. Desse modo os GET modelam SED em que somente os aspectos de sincronização são observados.

A Figura 2 exemplifica um GET, onde os círculos representam os lugares, os traços as transições, as flechas os arcos, os pontos as fichas e os números reais os tempos de atraso. Na Figura 2 é importante observar a existência de transições não condicionadas por nenhuma outra transição do sistema 
(transição $u$ na Figura 2) e transições que não condicionam nenhuma outra transição do sistema (transição $y$ na Figura 2). Estas transições são chamadas respectivamente de entradas e de saídas do sistema; as outras transições são chamadas de internas.

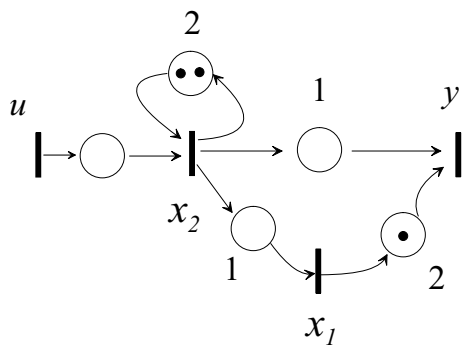

Figura 2: Exemplo de Grafo de Eventos Temporizado.

Pode-se associar a cada transição de um GET uma seqüência crescente de números inteiros $\{x(k)\}$, para $k=0,1,2, \ldots$, onde cada elemento da série representa o instante do $k$-ésimo disparo dessa transição. Suponha agora conhecida a seqüência associada às transições de entrada de um GET. A partir dessas informações é possível determinar as seqüências de disparo de todas as transições do GET. De fato, considere novamente o GET da Figura 2. É possível escrever as seguintes relações entre os instantes de disparo das transições:

$$
\begin{aligned}
x_{1}(k) & =1+x_{2}(k) \\
x_{2}(k) & =\max \left\{2+x_{2}(k-2) ; u(k)\right\} \\
y(k) & =\max \left\{2+x_{1}(k-1) ; 1+x_{2}(k)\right\}
\end{aligned}
$$

Renomeando o operador max como sendo $\oplus$ e o operador + como sendo $\otimes$, pode-se reescrever:

$$
\begin{aligned}
x_{1}(k) & =1 \otimes x_{2}(k) \\
x_{2}(k) & =2 \otimes x_{2}(k-2) \oplus u(k) \\
y(k) & =2 \otimes x_{1}(k-1) \oplus 1 \otimes x_{2}(k)
\end{aligned}
$$

As equações 2 acima ainda requerem que se especifique o estado inicial do sistema, ou seja, os valores de $x_{1}(k)$ e $x_{2}(k)$ para $k<0$. Nesse caso, arbitra-se que todos os disparos anteriores a $k=0$ ocorreram em $-\infty$. Conseqüentemente, a marcação inicial da rede já cumpriu o tempo de atraso de seus respectivos lugares e pode contribuir imediatamente para a habilitação de uma transição. Note que a marcação inicial promove deslocamentos na numeração dos disparos associados a uma transição. Tem-se, portanto, um sistema de equações recursivas lineares numa nova álgebra, denominada álgebra max-plus.

De modo geral, essa álgebra é um semi-anel idempotente (ou dióide), que é caracterizado por um conjunto e duas opera- ções ( $\operatorname{soma}$ e produto), notado $(D, \oplus, \otimes)$, tal que a soma seja associativa, comutativa e idempotente $(a \oplus a=a)$, e o produto seja associativo (mas não necessariamente comutativo) e distributivo à esquerda e à direita em relação à soma. Além disso, devem existir elementos neutros para ambas as operações, notados por $\varepsilon$ (elemento nulo) e por $e$ (elemento unitário), e o elemento nulo deve ser absorvente em relação ao produto. Isto é, $\forall a \in D, a \oplus \varepsilon=a, a \otimes e=a, a \otimes \varepsilon=\varepsilon$. Como na álgebra convencional, a multiplicação tem prioridade sobre a soma.

É imediato perceber que o conjunto $\mathbb{Z} \cup\{-\infty\}$ munido das duas operações $\oplus \equiv \max$ e $\otimes \equiv+$ é um dióide, no qual $\varepsilon=-\infty$ e $e=0$. Um dióide é completo se ele for fechado em relação a somas infinitas e se o produto for distributivo em relação a somas infinitas. A estrutura $(\mathbb{Z} \cup\{-\infty\} \cup\{\infty\}, \max ,+)$ é um dióide completo usualmente denominado Max-plus e notado por $\overline{\mathbb{Z}}_{\max }$. Uma importante operação, definida em qualquer dióide, é a operação estrela de Kleene, definida por $a^{*}=\bigoplus_{i \in \mathbb{N}} a^{i}$, com $a^{i}=a \otimes a^{(i-1)}$ e $a^{0}=e$. É imediato constatar que, para qualquer inteiro positivo $p,\left(a^{*}\right)^{p}=a^{*}$ e $\left(a^{*}\right)^{*}=a^{*}$.

O exemplo anterior utiliza o que se convenciona chamar de datadores, isto é, sequências crescentes $\{x(k)\}$ que representam as datas ou instantes de ocorrência dos disparos da transição $x$. De modo análogo ao que ocorre na teoria de sistemas contínuos, as manipulações das equações de um GET ficam facilitadas se forem utilizadas transformadas das sequências de datadores definidas de modo semelhante ao das clássicas transformadas Z. Define-se, portanto, a $\gamma$-transformada de uma seqüência $\{x(k)\}$ como sendo $x(\gamma)=\bigoplus_{k \in \mathbb{Z}^{+}} x(k) \gamma^{k}$, sendo $\gamma$ uma variável abstrata cujo significado é o de um operador "atraso em contagem", pois é imediato constatar que $y(\gamma)=\gamma x(\gamma) \Leftrightarrow\{y(k)\}=\{x(k-1)\}$. As equações 2 , submetidas à $\gamma$-transformada, resultam no seguinte sistema de equações algébricas:

$$
\begin{aligned}
x_{1}(\gamma) & =(1) x_{2}(\gamma) \\
x_{2}(\gamma) & =\left(2 \gamma^{2}\right) x_{2}(\gamma) \oplus u(\gamma) \\
y(\gamma) & =(2 \gamma) x_{1}(\gamma) \oplus(1) x_{2}(\gamma)
\end{aligned}
$$

É possível definir operações de soma e multiplicação entre séries formais do tipo $\bigoplus_{k \in \mathbb{Z}^{+}} x(k) \gamma^{k}$, utilizando-se para isso a soma e o produto adotados para $\overline{\mathbb{Z}}_{\max }$ combinados com os procedimentos usuais para somar e multiplicar séries formais. O conjunto de todas as séries formais em $\gamma$, munido dessas duas operações também é um dióide, isto é, satisfaz a todas as propriedades utilizadas para caracterizar um dióide, sendo denominado $\overline{\mathbb{Z}}_{\max } \llbracket \gamma \rrbracket$. Entretanto, esse dióide ainda não é adequado, considerando que admite a representação de seqüências decrescentes, o que não é desejável, pois o n- 
ésimo disparo não pode ocorrer após o $(n+1)$-ésimo disparo.

Para contornar esse problema define-se um novo conjunto, cujos elementos são sub-conjuntos (ou classes) de $\overline{\mathbb{Z}}_{\max } \llbracket \gamma \rrbracket$ contendo uma única série não-decrescente. Desse modo é possível estabelecer uma correspondência biunívoca entre esse novo conjunto e o conjunto das séries não-decrescentes em $\overline{\mathbb{Z}}_{\max } \llbracket \gamma \rrbracket$. Formalmente, dois elementos $a, b \in \overline{\mathbb{Z}}_{\max } \llbracket \gamma \rrbracket$ estarão na mesma classe se e somente se $a \gamma^{*}=b \gamma^{*} \mathrm{e}$ nesse caso $a$ e $b$ são ditos equivalentes. Por exemplo, a série $2 \gamma \oplus 1 \gamma^{2}$ (decrescente) é equivalente à série $2 \gamma$ pois $\left(2 \gamma \oplus 1 \gamma^{2}\right) \gamma^{*}=2 \gamma \gamma^{*} .{ }^{1}$ É importante destacar que o novo conjunto assim definido é um dióide porque é possível definir de maneira inequívoca operações de soma e multiplicação satisfazendo às propriedades usuais de dióides. Além disso, duas classes associadas a duas séries não-decrescentes quaisquer, terão como soma (ou produto) a classe associada à série que é a soma (ou produto) destas duas séries em $\overline{\mathbb{Z}}_{\text {max }} \llbracket \gamma \rrbracket$. No restante deste artigo o novo dióide constituído pelas classes acima descritas será denotado simplesmente por $\overline{\mathbb{Z}}_{\max } \llbracket \gamma \rrbracket$.

O resultado apresentado nas equações 3 pode ser generalizado. Para qualquer GET com $n$ transições internas (ou estados), $r$ entradas e $m$ saídas, pode-se escrever:

$$
\begin{aligned}
& x(\gamma)=A(\gamma) x(\gamma) \oplus B(\gamma) u(\gamma) \\
& y(\gamma)=C(\gamma) x(\gamma)
\end{aligned}
$$

sendo: $x \in \overline{\mathbb{Z}}_{\max }^{n} \llbracket \gamma \rrbracket, u \in \overline{\mathbb{Z}}_{\max }^{p} \llbracket \gamma \rrbracket, y \in \overline{\mathbb{Z}}_{\max }^{m} \llbracket \gamma \rrbracket, A \in$ $\overline{\mathbb{Z}}_{\max }^{n \times n} \llbracket \gamma \rrbracket, B \in \overline{\mathbb{Z}}_{\max }^{n \times p} \llbracket \gamma \rrbracket, C \in \overline{\mathbb{Z}}_{\max }^{m \times n} \llbracket \gamma \rrbracket$.

Outras representações, em outros dióides, podem ser utilizadas para descrever o comportamento dinâmico de um GET ver Baccelli et al. (1992). Em razão da grande semelhança entre todas essas possibilidades de representação, os SED descritos por GET e por equações em dióides são chamados simplesmente de sistemas Max-plus lineares.

A comparação entre dois elementos de um dóide é feita da seguinte maneira. Diz-se que $a \succeq b$ se e somente se $a=a \oplus$ $b$. Essa definição estabelece uma ordem parcial em qualquer dióide. Assim, dados dois elementos $a$ e $b$ é possível que não ocorra $a \succeq b$ nem $b \succeq a$, ou seja, $a$ e $b$ não são comparáveis. Esse é o caso, por exemplo, dos elementos $\gamma \oplus 3 \gamma^{2}$ e $\gamma \oplus 5 \gamma^{3}$ em $\overline{\mathbb{Z}}_{\text {max }} \llbracket \gamma \rrbracket$, cuja soma é $\gamma \oplus 3 \gamma^{2} \oplus 5 \gamma^{3}$.

A equação implícita $x=a x \oplus b$ aparece com freqüência em diversos problemas que envolvem sistemas Max-plus. Por exemplo, é o caso da equação 4. Para esse tipo de equação podem existir infinitas soluções, mas em geral a solução mínima (para o caso no qual se considera que a transição dis-

\footnotetext{
${ }^{1}$ Essa idéia pode ser também compreendida fazendo uma analogia com os números racionais. Por exemplo, $\frac{1}{2}$ é equivalente a $\frac{2}{4}$, pois $1 \times 4=2 \times 2$, ou seja, $\frac{a}{b}$ é equivalente a $\frac{c}{d}$ se, e somente se, $a d=b c$.
}

para imediatamente após a sua habilitação) apresenta maior interesse. Quando a equação está escrita num dióide completo, pode-se demonstrar (Baccelli et al., 1992) que sua solução mínima é dada por $x=a^{*} b$. Embora o modelo para um GET proposto na equação 4 seja constituído por um sistema de equações, o resultado acima pode ser aplicado, levando à expressão $y=C A^{*} B u=H u$ sendo as matrizes $\mathrm{A}$, B e C definidas na equação 4. A matriz $H=C A^{*} B \in \overline{\mathbb{Z}}_{\max }^{m \times p} \llbracket \gamma \rrbracket$ estabelece uma relação entre as saídas e as entradas do sistema e é por isso denominada matriz de transferência do GET. Por exemplo, a matriz de transferência do GET da Figura 2 é dada por $H(\gamma)=1 \oplus 3 \gamma\left(2 \gamma^{2}\right)^{*}$, sendo obviamente escalar (nesse caso chamada de função de transferência).

No contexto Max-plus é oportuno definir a entrada impulsiva como sendo uma seqüencia infinita de disparos em $t=0$, isto é, $\{u(k)\}=\{0,0,0, \ldots\}$, cuja transformada é $u(\gamma)=$ $e \oplus \gamma \oplus \gamma^{2} \oplus \gamma^{3} \oplus \ldots=\gamma^{*}$. Considerando a noção de equivalência introduzida anteriormente e o fato de que $\gamma^{*} \gamma^{*}=e \gamma^{*}$ conclui-se que $\gamma^{*}=e$, isto é, como no caso dos sistemas contínuos, a $\gamma$-transformada da entrada impulsiva é o elemento unitário. Para $u(\gamma)=e$ tem-se $y(\gamma)=H(\gamma)$ e portanto a resposta ao impulso de um sistema Max-plus linear é a anti-transformada da própria função de transferência. Retomando o exemplo anterior, obtém-se para a entrada impulsiva $y(\gamma)=1 \oplus 3 \gamma\left(2 \gamma^{2}\right)^{*}=1 \oplus 3 \gamma \oplus 5 \gamma^{3} \oplus 7 \gamma^{5} \oplus 9 \gamma^{7} \ldots=1 \oplus$ $3 \gamma \oplus 3 \gamma^{2} \oplus 5 \gamma^{3} \oplus 5 \gamma^{4} \oplus 7 \gamma^{5} \oplus 7 \gamma^{6} \oplus 9 \gamma^{7} \oplus 9 \gamma^{8}$. . . A partir da Figura 2 é possível constatar que, para a entrada impulsiva, a saída é de fato a anti-transformada desta expressão, isto é, $\{y(k)\}=\{1,3,3,5,5,7,7, \ldots\} \quad(k=0,1,2,3, \ldots)$.

De modo geral convenciona-se que $y(k)=\epsilon$, se $k<0$ (isto é, os disparos ocorridos antes do zero-ésimo disparo, por convenção, se dão em $t=-\infty$ ).

A Figura 3 mostra graficamente a trajetória de disparos $y(k)$. Os pontos marcados com $(\times)$, interligados pela linha trace-

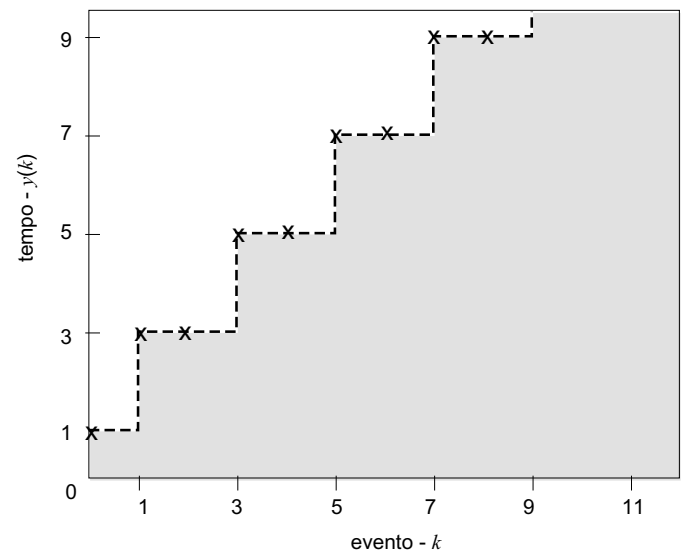

Figura 3: Trajetória mínima de disparo $y(k)$ para entrada impulsiva. 
jada correspondem à solução mínima, ou seja, aos mínimos instantes de disparo. O evento de número zero (primeiro disparo) ocorre a partir de $\mathrm{t}=1$; os eventos de números 1 e 2 (segundo e terceiro disparos respectivamente) ocorrem a partir de $\mathrm{t}=3$ e assim por diante. Na Figura 3, a área escura corresponde à região onde é impossível a ocorrência de qualquer disparo.

\subsection{Teoria de residuação}

Como é usual em determinadas classes de problemas de controle, as formulações discutidas na próxima seção envolvem a inversão de funções, isto é, a solução em $x$ de equações do tipo $y=f(x)$. Diferentemente do caso da álgebra tradicional a solução desse tipo de equação nos dióides pode apresentar um número infinito de soluções ou nenhuma solução. A Teoria de Residuação (Blyth e Janowitz, 1972) se ocupa justamente da solução desse problema em conjuntos parcialmente ordenados. A seguir, alguns resultados fundamentais relativos a essa teoria serão apresentados.

Um mapeamento $f$ de um conjunto ordenado $\mathcal{D}$ em um conjunto ordenado $\mathcal{C}$ é dito ser isotônico se $\forall a, b \in \mathcal{D}, \quad a \succeq$ $b \Longrightarrow f(a) \succeq f(b)$. Considere o mapeamento isotônico $f: \mathcal{D} \rightarrow \mathcal{E}$, sendo $\mathcal{D}$ e $\mathcal{E}$ dióides completos. Se existir um elemento máximo $x_{o p}$ do conjunto $\{x \mid y \succeq f(x), x \in$ $\mathcal{D}, y \in \mathcal{E}\}$, diz-se que $x_{o p}$ é o resíduo do mapeamento $f$ em $y$. Se $f$ tiver um resíduo em qualquer ponto $y \in \mathcal{E}$, o mapeamento $f$ é dito residuável, sendo o resíduo denotado por $f^{\sharp}(y)$. Um mapeamento $f$ é dito semi-contínuo inferior se para qualquer subconjunto (finito ou infinito) $X \subset \mathcal{D}$ temse $\bigoplus_{x \in X} f(x)=f\left(\bigoplus_{x \in X} x\right)$. Um mapeamento isotônico $f$ é residuável se e somente se $f(\varepsilon)=\varepsilon$ e $f$ é semi-contínuo inferior (Baccelli et al., 1992).

De fundamental importância na álgebra de dióides é a residuação do produto, pois formaliza a operação de "divisão"nessa álgebra. Basicamente, a solução de equações do tipo $a x=b$ esbarra em diversas dificuldades. Por exemplo, essa igualdade pode não ter solução ou pode ter várias soluções. No entanto, a inequação $a x \preceq b$ sempre tem solução $(x=\epsilon) \mathrm{e}$ até mesmo um conjunto de soluções, que é parcialmente ordenado. Assim, dentre as soluções desse conjunto, a solução obtida por residuação é de especial interesse, pois é a solução máxima. Isso é formalizado a seguir.

As funções $L_{a}(x)=a \otimes x$ e $R_{a}(x)=x \otimes a$, por satisfazerem as hipóteses acima (i.e são isotônicas, semi-contínuas inferiores e $L_{a}(\epsilon)=R_{a}(\epsilon)=\epsilon$ ), são residuáveis em qualquer dióide completo, sendo seus resíduos denotados respectivamente por $L_{a}^{\sharp}=a \phi x$ ("divisão à esquerda" por $a$ ) e $R_{a}^{\sharp}=x \phi a$ ("divisão à direita" por $a$ ). Em geral, essas duas residuações conduzem a resultados diferentes, exceto obvia- mente quando o produto é comutativo. No caso particular do dióide $\overline{\mathbb{Z}}_{\text {max }}$ tem-se $L_{a}^{\sharp}=R_{a}^{\sharp}=x-a$ (operação de subtração da álgebra tradicional).

No dióide $\overline{\mathbb{Z}}_{\max } \llbracket \gamma \rrbracket$ apresentado na seção anterior, é possível mostrar (Baccelli et al., 1992) que para quaisquer duas séries $u(\gamma)=\bigoplus_{k \in \mathbb{Z}^{+}} u(k) \gamma^{k}$ e $v(\gamma)=\bigoplus_{k \in \mathbb{Z}^{+}} v(k) \gamma^{k}$ tem-se:

$$
v(\gamma) \phi u(\gamma)=\bigoplus_{k \in \mathbb{Z}^{+}} \bigwedge_{l \geq k}(v(l-k) \phi u(l)) \gamma^{k} .
$$

A expressão $\bigwedge_{l \geq k}(v(l-k) ф u(l))$ corresponde ao coeficiente de $\gamma^{k}$, sendo $(\bigwedge)$ o operador $\min ^{2}$ e $v(l-k) \phi u(l)=$ $u(l)-v(l-k)$ (operação de subtração da álgebra tradicional). Convém ainda relembrar a definição de residuação, que assegura que $y(\gamma)=v(\gamma) \oint u(\gamma)$ é a maior série tal que $v(\gamma) \otimes y(\gamma) \preceq u(\gamma)$. Maiores detalhes sobre a residuação de séries são apresentados por Baccelli et al. (1992). A seguir, alguns exemplos de residuação no dióide $\overline{\mathbb{Z}}_{\max } \llbracket \gamma \rrbracket$ são apresentados:

1. $\gamma \phi\left(\gamma \oplus 2 \gamma^{2}\right)=\gamma^{-1}\left(\gamma \oplus 2 \gamma^{2}\right)=e \oplus 2 \gamma$.

2. $5 \gamma \phi \gamma^{3}=\varepsilon$. Observar que são considerados apenas o elementos realizáveis de $\overline{\mathbb{Z}}_{\max } \llbracket \gamma \rrbracket$, caso considerássemos também os não realizáveis, teríamos $5 \gamma \phi \gamma^{3}=$ $(-5) \gamma^{2}$, isso significa que o terceiro disparo ocorre em um instante de tempo negativo (no caso, ocorre em -5 ).

3. $5 \gamma \phi\left(\gamma^{3} \oplus 7 \gamma^{4}\right)=(-5) \gamma^{-1}\left(\gamma^{3} \oplus 7 \gamma^{4}\right)=\varepsilon \oplus(-5) 7 \gamma^{3}=$ $2 \gamma^{3}$.

4. $y(\gamma)=(1 \oplus 2 \gamma) \phi\left(1 \oplus 7 \gamma^{2}\right)$. Nesse caso, aplicase a fórmula geral apresentada na Equação 5, sendo $u(\gamma)=1 \oplus 7 \gamma^{2}$ e $v(\gamma)=1 \oplus 2 \gamma$. Lembrando da definição de classe de equivalência que $u(\gamma)=\gamma^{*} u(\gamma)$ temse que $u(\gamma)$ equivale a $\left(1 \oplus 1 \gamma \oplus 7 \gamma^{2} \oplus 7 \gamma^{3} \oplus 7 \gamma^{4} \ldots\right)$ e que $v(\gamma)$ equivale a $\left(1 \oplus 2 \gamma \oplus 2 \gamma^{2} \oplus 2 \gamma^{3} \ldots\right)$. Dessa maneira, mostra-se que $y(\gamma)=\gamma \oplus 5 \gamma^{2} \oplus 5 \gamma^{3} \oplus 5 \gamma^{4} \ldots=$ $\left(\gamma \oplus 5 \gamma^{2}\right) \gamma^{*}$, e, portanto, pela relação de equivalência, obtém-se $y(\gamma)=\gamma \oplus 5 \gamma^{2}$.

Cálculos de resíduos mais complexos no dióide $\overline{\mathbb{Z}}_{\max } \llbracket \gamma \rrbracket$ podem ser feitos utilizando o pacote computacional SCILAB com as rotinas disponibilizadas por Lhommeau e Hardouin (2003).

De maneira geral, se $\mathcal{D}$ é um dióide completo, então $\mathcal{D}^{n \times n}$ também é um dióide e as operações $L_{A}(X)=A \otimes X$ e $R_{A}(X)=X \otimes A$ também são residuáveis, sendo $A$ e $X$ matrizes $n \times n$ com coeficientes em $\mathcal{D}$. É possível mostrar que a matriz $L_{A}^{\sharp}=A \phi X$ pode ser calculada por:

\footnotetext{
${ }^{2} a \wedge b$ é o maior elemento menor do que $a$ e menor do que $b$.
} 


$$
(A \nmid X)_{i j}=\bigwedge_{l=1}^{n} A_{l i} \nmid X_{l j},
$$

Novamente nesse caso utiliza-se o operador min, sendo a operação de residuação entre elementos das matrizes aquela definida para os elementos do dióide $\mathcal{D}$. Caso se trate do dióide $\overline{\mathbb{Z}}_{\text {max }}$ esta operação será uma subtração. Caso $\mathcal{D}$ coincida com o dióide $\overline{\mathbb{Z}}_{\max } \llbracket \gamma \rrbracket$ esta operação será dada pela Equação 5. De modo análogo, define-se o resíduo $X \phi A$ substituindo na expressão acima o operador " $\$$ "pelo operador " $\phi$ "e invertendo as posições dos coeficientes das matrizes $A$ e $X$.

Algumas fórmulas úteis envolvendo o resíduo das operações $L_{a}(x)$ e $R_{a}(x)$ e a operação estrela de Kleene são apresentadas a seguir:

$$
\begin{aligned}
a(a ф x) & \preceq x \\
a(a ф(a x)) & =a x \\
a ф a & =(a ф a)^{*} \\
\left(a^{*}\right)^{2} & =a^{*} \\
a(b a)^{*} & =(a b)^{*} a
\end{aligned}
$$

A título de ilustração e de simplificação dos resultados apresentados na literatura (Baccelli et al., 1992), notadamente para a fórmula 8 , são apresentadas a seguir novas demonstrações para as fórmulas 6 a 8 . O ponto chave para a obtenção dos resultados é ter em mente a definição de que $a \oint y$ é o maior elemento $x$ que satisfaz à desigualdade $a x \preceq y$.

- A fórmula 6 advém diretamente da definição da residuação, pois , conforme observado anteriormente, $z_{o p}=$ $a \oint x$ é o maior elemento que satisfaz à desigualdade $a z \preceq x$.

- Para a fórmula 7, sabe-se que $z_{o p}=a \phi(a x)$ é o maior elemento $z$ que satisfaz $a x \succeq a z, \log 0, a x \succeq a z_{o p}$. Como $z=x$ também satisfaz essa desigualdade, $z_{o p} \succeq$ $x$ e, portanto, em decorrência da isotonia da multiplicação, $a z_{o p} \succeq a x$. Dessa forma, $a x \succeq a z_{o p}$ e $a z_{o p} \succeq a x$ o que resulta em $a x=a z_{o p}$. Em outras palavras, se $z=x$ satisfaz $a z=a x$, então $z=a \phi(a x)$ também satisfaz essa equação.

- $z_{o p}=a \oint a$ é a máxima solução da inequação $a \succeq a z$ $\mathrm{e}$, portanto, $a \succeq a z_{o p}$. Assim, pela isotonia da multiplicação, $a \succeq a z_{o p} \succeq a z_{o p}^{2} \succeq \ldots \succeq z_{o p}^{k} \succeq \ldots$. Somando todos os termos dessas desigualdades ${ }^{3}$, obtémse $a \succeq a\left(e \oplus z_{o p} \oplus z_{o p}^{2} \oplus \ldots \oplus z_{o p}^{k} \oplus \ldots\right)=a z_{o p}^{*}$. Portanto $z_{o p}^{*}$ satisfaz a inequação e, por definição temse que $z_{o p} \succeq z_{o p}^{*}$. Mas $z_{o p}^{*}=e \oplus z_{o p} \oplus z_{o p}^{2} \oplus \ldots \succeq z_{o p}$,

\footnotetext{
${ }^{3}$ Relembrar que $a \succeq b \Leftrightarrow a \oplus b=a$
}

$\operatorname{logo} z_{o p}^{*}=z_{o p}$. Em suma, esse resultado significa que $a \oint a$ é maior ou igual a qualquer uma das suas potências. Esse resultado é central para a síntese de controladores.

\section{CONTROLE POR MODELO DE REFE- RÊNCIA}

O problema de controle de um GET é usualmente formulado tendo como objetivo obter trajetórias para as transições de entrada as mais tardias possíveis, sem entretanto violar especificações de trajetórias de disparo mínimas para as transições de saída. Esta formulação do problema ganha apelo intuitivo ao se considerar a estratégia "just-in-time" em sistemas de manufatura. Nela deseja-se operar a planta de modo que os estoques (de matéria-prima) em seu interior sejam mínimos, mas garantindo que sua produção atenda à demanda pré-especificada, ou seja, que as datas devidas ("duedates") para o produtos acabados sejam cumpridas. Um caso particular de importância é aquele em que se deseja, do ponto de vista da saída, que a planta se comporte como se não houvesse limitações de matéria-prima, trabalhando portanto com sua máxima capacidade de produção. Para que isso aconteça não é necessário, em geral, que os recursos estejam todos disponíveis ao se iniciar a produção, mas sim no momento exato em que puderem ser processados. Do ponto de vista de um GET isso significa que as trajetórias das transições de saída devem ser idênticas àquelas obtidas quando as transições de entrada são impulsivas, isto é, em $t=0$ as transições de entrada disparam infinitas vezes. Para que essas saídas sejam obtidas não é necessário entretanto (em geral) que as entradas sejam impulsivas. A questão é portanto, obter a trajetória mais tardia possível na entrada, sem que a trajetória da saída tenha seu desempenho alterado.

Considere agora um GET modelado em $\overline{\mathbb{Z}}_{\max } \llbracket \gamma \rrbracket$, resultando em expressões do tipo dado pelas equações 4 . A matriz de transferência do sistema é dada por $H=C A^{*} B$, de modo que $y=H u$. Seja também $z \in \overline{\mathbb{Z}}_{\max } \llbracket \gamma \rrbracket$ uma trajetória que represente o desempenho aceitável para a transição de saída. É imediato constatar que dadas duas trajetórias $a, b \in \overline{\mathbb{Z}}_{\max } \llbracket \gamma \rrbracket$, se $a \succeq b$ então a trajetória $a$ é mais lenta que a trajetória $b$. O problema de obter a trajetória de controle ótima é então equivalente ao de obter a máxima trajetória $u$ tal que $z \succeq y=H u$. Como a função $H u$ é residuável, o resultado é $u_{o p}=H ф z$. Em particular, se se deseja que a planta opere em sua máxima taxa de produção, a trajetória $z$ deve ser igual a $H u$ sendo $u$ a entrada impulsiva. Conseqüentemente, para o caso de produção máxima, $z=H \mathrm{e}$ portanto $u_{\text {otimo }}=H \phi H$. Deve-se observar que a relação $H ф H \succeq e$ é sempre verificada de modo que a entrada ótima é, como esperado, mais lenta que a entrada impulsiva.

Uma abordagem usual em projeto de sistemas de controle é a alteração da matriz de transferência da planta através de 
seu acoplamento a um outro sistema, usualmente chamado de compensador. No caso de sistemas Max-plus, dois aspectos devem ser levados em conta, ao se considerar essa abordagem.

Em primeiro lugar deve-se determinar sobre que transições ocorrerá a ação de controle, isto é, quais são as transições "controláveis" no sentido de permitirem sua inibição por um dispositivo externo à planta. Em relação a esse aspecto, duas alternativas serão consideradas neste artigo. A primeira delas parte do pressuposto de que apenas a variável de entrada $u(\gamma)$ é controlável (no sentido definido acima) cabendo portanto calculá-la a partir de alguma outra informação externa. Nos sistemas de manufatura, por exemplo, esta informação externa pode estar associada à disponibilidade de recursos no ambiente externo à planta. A variável $v(\gamma)$ será associada a esta informação, sendo chamada de "sinal de referência". A segunda alternativa é considerar um conjunto arbitrário de transições da planta como sendo controláveis. Nesse caso, a transição de entrada $(u(\gamma))$ permanece sendo aquela que sofre as influências do ambiente externo à planta.

Em segundo lugar deve-se determinar qual deverá ser o desempenho do sistema sob a ação do controlador. De modo geral, é possível calcular a função de transferência entre as saídas do sistema e as variáveis que sofrem influências externas ao sistema $(u(\gamma)$ ou $v(\gamma))$ levando-se em conta a presença do compensador. O desempenho do sistema será então especificado a partir da determinação de uma função de transferência de referência, notada por $G_{r e f}(\gamma)$ e também denominada "modelo de referência", com a mesma dimensão da função de transferência do sistema. Determinados estes dois aspectos, o compensador deve então ser calculado de modo a ser máximo (i.e. o controlador deve retardar ao máximo sua saída), mas garantindo que o comportamento do sistema sob a ação do controle permaneça limitado superiormente pelo modelo de referência. Em outras palavras, esse comportamento não pode ser mais lento que o imposto pelo modelo de referência.

Observa-se que maximizar o controlador não é exatamente o mesmo que maximizar o sinal de controle, pois ainda pode existir um sinal de controle maior, mas que eventualmente não pode ser obtido pelo controlador máximo. Entretanto, de modo geral, quanto "maior" o controlador, maior o atraso ele provoca entre sua entrada e sua saída. Em muitos casos pode-se provar que esta estratégia leva a resultados iguais aos obtidos pela otimização do sinal de controle (Maia, 2003).

Portanto o projeto de controladores é feito de modo a satisfazer a restrição imposta pelo modelo de referência maximizando os controladores e por isso retardando os disparos das transições sobre as quais se dá a ação de controle. Hardouin (2004) apresenta uma compilação sobre essa abordagem. Na próxima seção, algumas alternativas serão consideradas.

\section{SÍNTESE DE CONTROLADORES}

Na seção anterior, o problema de controle por modelo de referência foi estabelecido na sua forma geral. Nessa seção, são apresentadas as estruturas de controle utilizadas. A précompensação da Subseção 4.1 e a realimentação de saída da Subseção 4.2 têm sido amplamente estudadas na literatura. Já a estratégia P-F da Subseção 4.3 e o controle multivariável generalizado da Subseção 4.4 representam as principais contribuições deste artigo.

\subsection{Pré-compensação}

Uma primeira estratégia para o controle por modelo de referência consiste na utilização de pré-compensação. Nesse caso, o problema de controle é encontrar um précompensador tal que a entrada do sistema seja maximizada respeitando a restrição de que a função de transferência do sistema controlado satisfaça as limitações impostas pelo modelo de referência. Essa estratégia é ilustrada na Figura 4(Cottenceau, 1999).

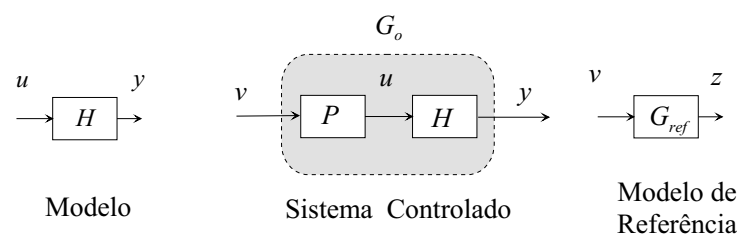

Figura 4: Controle por pré-compensação

Formalmente, pode se mostrar que a solução para o problema equivale a calcular o maior pré-compensador $P$ tal que $H P \preceq G_{r e f}$. Como a função $f(x)=a \otimes x$ é residuável (Baccelli et al., 1992), mostra-se que a maior solução dessa inequação, denotada por $P_{o p}$, é dada por:

$$
P_{o p}=H \phi G_{r e f} .
$$

Essa estratégia de controle em malha aberta resulta na ação de controle $u=P_{o p} v$ que assegura sempre desempenho ótimo do sistema. Contudo, não se assegura a estabilidade ${ }^{4}$ robusta do sistema em relação a variações paramétricas, isto é, variações dos parâmetros temporais da planta podem comprometer a estabilidade do sistema. De fato, considere um sistema Max-plus linear tal que $H=1(7 \gamma)^{*}$. Esse sistema modela uma máquina que opera em ciclos com uma taxa de

\footnotetext{
${ }^{4} \mathrm{O}$ conceito de estabilidade aqui está relacionado com o número de recursos de um lugar. Diz-se que um sistema é estável se o número de recursos de um lugar é sempre limitado, ou seja, não pode crescer indefi nidamente
} 
produção igual a uma peça a cada 7 unidades de tempo e com um atraso de transporte para disponibilização final igual a 1 unidade de tempo. Esse modelo é mostrado em linhas pontilhadas na Figura 5(a). Considere também que a restrição de demanda é dada por um modelo de referência tal que $G_{r e f}=H$, ou seja, o objetivo é preservar a função de transferência em malha aberta maximizando a entrada do sistema. Dessa forma, dentro de um contexto de gestão de recursos "just-in-time", preserva-se a máxima taxa de produção do sistema retardando ao máximo possível a entrada de matéria prima a ser processada. Conforme visto anteriormente, o problema se resume a encontrar o maior controlador $P$ tal que $H P \preceq G_{\text {ref }}$. A solução para esse problema é dada pela Teoria de Residuação $P=H ф H=(7 \gamma)^{*}$.

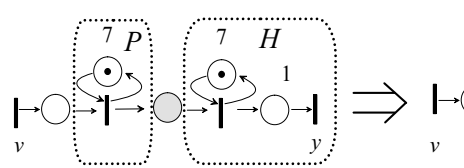

(a)

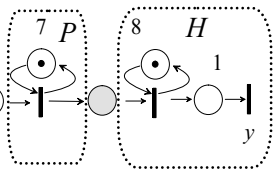

(b)
Figura 5: Problema de estabilidade na pré-compensação.

É importante observar que qualquer outro modelo de referência pode ser utilizado. Por exemplo, adotando-se $G_{r e f}=$ $1(8 \gamma)^{*}$, o pré-compensador será dado por $P=(8 \gamma)^{*}$.

Voltando ao caso em que $G_{r e f}=H\left(\right.$ e $\left.P=(7 \gamma)^{*}\right)$, podese observar, a partir da Figura 5, que o sistema é sensível a variações paramétricas, o que pode ocasionar problemas de instabilidade. No presente exemplo, se a temporização do ciclo aumentar (por exemplo por algum problema de manutenção da máquina) o pré-compensador se torna mais rápido do que o sistema e o número de recursos no lugar situado entre $P$ e $H$ cresce ilimitadamente.

\subsection{Realimentação de saída}

Sabe-se da Teoria de Controle de Sistemas Lineares Contínuos que a realimentação torna o sistema controlado menos sensível a variações paramétricas da planta, contudo, o sistema em malha fechada pode se tornar instável. Nos SED Max-plus lineares, a estratégia de controle via realimentação sempre favorece a estabilidade, conforme mostrado por Maia (2003). Nesse sentido, para se contornar o problema de instabilidade, assegurando um sistema mais robusto (menos sensível a variações paramétricas), recomenda-se a utilização da estratégia de realimentação para o controle por modelo de referência. $\mathrm{O}$ exemplo abaixo mostra como a realimentação pode favorecer a estabilidade do sistema.

Considere o mesmo sistema do exemplo anterior, ou seja,
$H=1(7 \gamma)^{*}$ com realimentação dada por $F=6 \gamma(7 \gamma)^{*}$. O sistema em malha fechada é mostrado na Figura 6(a). Mostra-se, conforme será visto a seguir, que essa realimentação preserva a função de transferência $H$ do sistema. É interessante observar que a estabilidade do sistema em malha fechada é independente das variações paramétricas, pois a admissão de matéria prima é condicionada à saída de produtos do sistema. De fato, vê-se na Figura 6(b) que o aumento da temporização do ciclo da máquina não acarreta explosão do número de recursos no sistema (na verdade eles permanecem constantes).

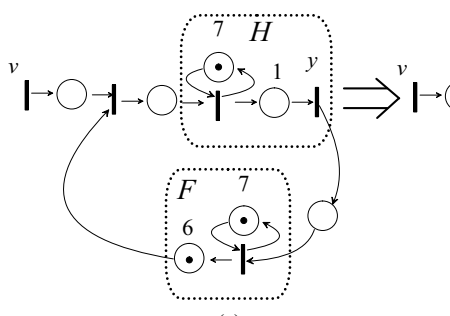

(a)

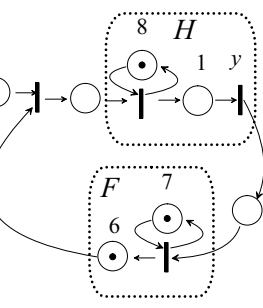

(b)
Figura 6: Estabilidade robusta da realimentação

A Figura 7 ilustra de maneira geral a estrutura de controle com realimentação da saída. Na Figura 7, $H$ é função de transferência da planta, $F$ é o controlador e $u$ e $y$ são respectivamente a entrada e a saída do sistema; $G_{\text {ref }}$ é o modelo de referência e $v$ e $z$ são respectivamente a entrada de referência (disponibilidade de matéria prima) e $z$, a saída do modelo de referência.

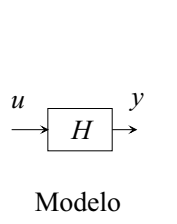

(1)

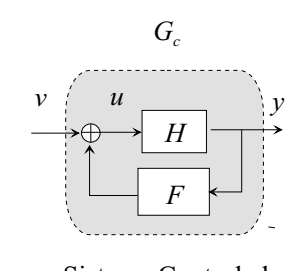

Sistema Controlado
Figura 7: Controle por realimentação

A partir da análise Figura 7, obtém-se as seguintes equações:

$$
\begin{aligned}
& u=v \oplus F y, \\
& y=H u=H F y \oplus H v .
\end{aligned}
$$

A solução da Equação 13 é dada na Seção 2.1, ou seja:

$$
y=(H F)^{*} H v \text {. }
$$

Dessa forma, $u=\left(e \oplus F(H F)^{*} H\right) v$. A Equação 10 garante que $(H F)^{*} H=H(F H)^{*}$. Portanto $u=(e \oplus$ 
$\left.F H(F H)^{*}\right) v=\left(e \oplus F H \oplus(F H)^{2} \oplus \ldots\right) v=(F H)^{*} v$. Consequientemente:

$$
u=(F H)^{*} v .
$$

O problema de controle para a estratégia de realimentação consiste então em maximizar $u=(F H)^{*} v$ tal que a função de transferência em malha fechada $H(F H)^{*}$ seja menor ou igual ao modelo de referência $G_{r e f}$. Em decorrência da isotonia dos operadores de adição e de multiplicação do dióide, esse problema equivale a encontrar a maior realimentação $F$ tal que $H(F H)^{*}$ seja menor ou igual a referência $G_{r e f}$. Formalmente, o que se procura é a maior solução para a inequação $H(F H)^{*} \preceq G_{r e f}$. Esse problema não tem solução máxima para qualquer modelo de referência, pois a função $G(X)=H(X H)^{*}$ não é sempre residuável. Contudo, a solubilidade pode ser assegurada se restrições paramétricas são impostas ao modelo de referência. Cottenceau et al. (2001) demonstraram que se $G_{r e f}$ é da forma $A^{*} H$ ou da forma $H B^{*}$ (sendo $A$ e $B$ matrizes de transferência quaisquer de dimensões apropriadas), então existe um compensador $F$ que maximiza a inequação anterior. Esses resultados foram generalizados por Lüders e Santos-Mendes (2002) considerando o problema em que somente parte das transições de estado estão disponíveis para observação e controle (acesso parcial aos estados). Dentre os resultados obtidos, são apresentadas condições suficientes para a existência de solução para o problema proposto (Lüders, 2001). Mais recentemente, Maia et al. (2003) propuseram uma estrutura em malha fechada que utiliza pré-compensação e realimentação. Esses resultados serão apresentados a seguir.

\subsection{Estratégia P-F}

Conforme explicado na Seção 3, as estratégias baseadas em realimentação, embora favoreçam a estabilidade, são limitadas no sentido em que o modelo de referência deve satisfazer certas condições restritivas. Já as baseadas em précompensação garantem desempenho ótimo para qualquer modelo de referência mas não garantem estabilidade. A estratégia de controle a ser apresentada é baseada na utilização simultânea da pré-compensação e realimentação. A principal vantagem dessa abordagem é que ela garante otimalidade em relação aos estoques que alimentam a planta para qualquer modelo de referência escolhido. Além disso, sob determinadas condições, garante-se que sistema controlado é sempre estável (Maia et al., 2003). As duas principais vantagens dessa abordagem em relação à realimentação, como proposto por Cottenceau et al. (2001), são: a ação de controle ótima é sempre máxima (igual a $P_{o p} v$ ) e não há nenhuma restrição na escolha do modelo de referência. A Figura 8 ilustra a abordagem proposta.

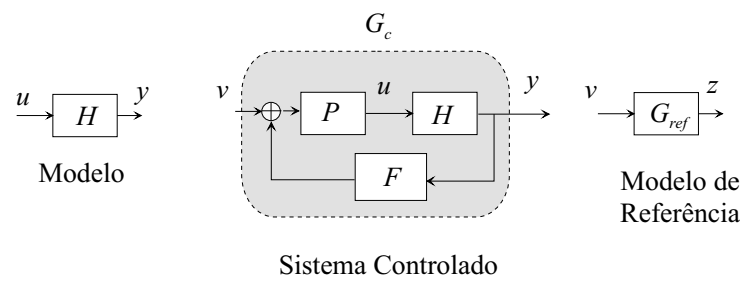

Figura 8: Estratégia de controle P-F

Dessa forma, escrevem-se as seguintes equações:

$$
\begin{array}{r}
u=P v \oplus P F y, \\
y=(H P F) y \oplus H P v .
\end{array}
$$

Usando os resultados da Seção 2.1 e a Equação 10, obtêm-se as equações que relacionam $u$ e $y$ a $v$

$$
\begin{gathered}
y=G_{c} v=(H P F)^{*} H P v=H P(F H P)^{*} v, \\
u=G_{u v} v=(P F H)^{*} P=P(F H P)^{*} v,
\end{gathered}
$$

sendo $G_{c}$ e $G_{u v}$ as funções de transferência em malha fechada entre $y$ e $v$ e entre $u$ e $v$ respectivamente.

O problema de controle pode então ser enunciado da seguinte maneira. Dado um sistema max-plus com função de transferência $H$, quais são as matrizes do controlador $P$ e $F$ que asseguram a maior função de transferência entre u e $v$, i.e. $G_{u v}$, tal que $G_{C} \preceq G_{r e f}$ ? Num contexto de sistema de produção, conforme já discutido anteriormente, considerando a política "just-in-time", o que se busca é um controlador que satisfaça a especificação da demanda, $G_{c} \preceq G_{r e f}$, atrasando o máximo possível a entrada de matéria prima a ser processada. Formalmente, esse problema pode ser enunciado como:

$$
\begin{gathered}
\sup _{P, F} G_{u v}=P(F H P)^{*} \\
\text { t.q. } G_{c}=H P(F H P)^{*} \preceq G_{r e f} .
\end{gathered}
$$

O problema apresentado em 20 tem sempre $P=[\varepsilon]_{p \times p}$ como uma solução independentemente da escolha de $F$, o que significa que o conjunto de soluções é não-vazio. Além disso, é fácil ver que as estratégias que usam exclusivamente pré-compensação (fazendo com que $F=[\varepsilon]_{p \times m}$ ) ou exclusivamente realimentação (fazendo-se $P=I_{p \times p}$ sendo $I_{p \times p}$ uma matriz identidade em dióide) são caso particulares da estrutura de controle proposta.

O problema de otimização proposto em (20) tem sempre solução ótima que é obtida com:

$$
\begin{aligned}
P_{o p} & =H \phi G_{r e f} . \\
F_{o p} & =\left(H P_{o p}\right) \phi\left(H P_{o p}\right) \phi\left(H P_{o p}\right) .
\end{aligned}
$$


A prova desse resultado é dada por Maia et al. (2003).

É importante observar que diferentemente da estrutura com realimentação proposta por Cottenceau (1999), que utiliza somente o controlador F, a presente abordagem não impõe nenhuma restrição na escolha do modelo de referência.

\section{Propriedades da estrutura P-F}

1. A solução dada pelas expressões 21 e 22 sempre assegura que $G_{u v}=H \phi G_{r e f}$ e $G_{c}=H P_{o p}$.

2. Se existir uma matriz $D$ tal que $G_{r e f}=H D$ então a solução ótima para a estrutura de controle proposta garante que $G_{c}=G_{r e f}$.

3. Se o objetivo é preservar a resposta impulsiva do sistema, isto é, $G_{r e f}=H$, a solução ótima é obtida se $P_{o p}=H ф H$ e $F_{o p}=H ф H \phi H$.

A primeira propriedade significa que a solução proposta sempre garante que as funções de transferência em malha fechada $G_{u v}$ e $G_{c}$ são iguais aos seus limitantes superiores, ou seja, a solução obtida é a melhor possível; a segunda condição significa que é sempre possível fazer com que sistema em malha fechada se comporte como o modelo de referência (total matching); finalmente, a condição número 3, significa que a condição ótima obtida pela realimentação simples, conforme proposto por Cottenceau et al. (1999), pode ser ainda melhorada com a adição de um pré-compensador.

Considere a planta mostrada na Figura 9 como exemplo ilustrativo de aplicação das idéias propostas nesta seção.

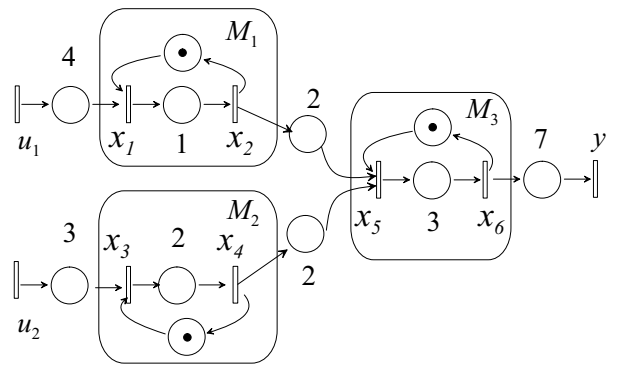

Figura 9: Sistema de manufatura com 3 máquinas.

Essa planta representa um pequeno sistema de manufatura com 3 máquinas $\left(M_{1}, M_{2}\right.$ e $\left.M_{3}\right)$. Nele as matérias primas são processadas pelas máquinas $M_{1}$ e $M_{2}$ (cujas datas de entrada são dadas por $u_{1}$ e $u_{2}$ respectivamente) e os subprodutos dessas máquinas são agrupados e trabalhados pela máquina $M_{3}$, resultando em um produto final cuja data de produção é dada por $y$. Segundo os resultados da Seção 2.1, mostra-se que a equação que relaciona a entrada e a saída da planta é dada por:

$$
y=\left[17(3 \gamma)^{*} 17(3 \gamma)^{*}\right] u .
$$

Dessa forma a função de transferência da planta é dada por $H=\left[17(3 \gamma)^{*} 17(3 \gamma)^{*}\right]$. Nesse exemplo, a máquina $M_{3}$ é o gargalo do sistema e sua taxa máxima de produção é uma peça a cada três unidades de tempo. Analiticamente, isto é confirmado pela presença do fator $(3 \gamma)^{*}$ nos dois elementos da matriz $H$. Se o objetivo é fazer com que o sistema tenha o maior perfil de produção possível, o modelo de referencia deve ser escolhido como sendo $G_{r e f}=H$, em outra palavras, o modelo de referência deve ser igual à resposta do sistema para um estoque infinito. A solução ótima é obtida através das equações 21 e 22 , o que resulta em: ${ }^{5}$

$$
P_{o p}=(3 \gamma)^{*}\left(\begin{array}{ll}
e & e \\
e & e
\end{array}\right) \text { e } F_{o p}=\left(1 \gamma^{6}\right)(3 \gamma)^{*}\left(\begin{array}{ll}
e & e
\end{array}\right)
$$

Finalmente, o sistema controlado é mostrado na Figura 10. Pode-se ver que o sistema em malha fechada é fortemente conexo. Esse fato faz com que a admissão de matérias primas seja condicionada à saída de produtos $\mathrm{e}$, dessa forma, $\mathrm{o}$ número de recursos no sistema em um dado instante é sempre limitado; portanto, o sistema é sempre estável.

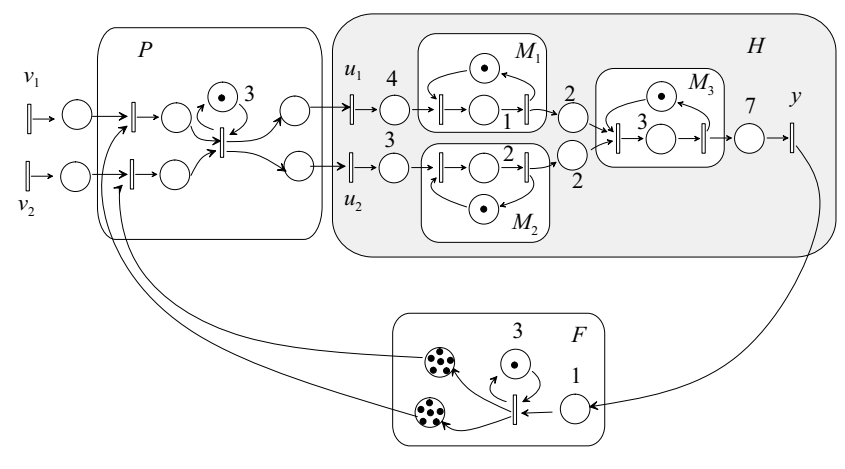

Figura 10: Sistema de manufatura controlado

Comparando o desempenho do sistema controlado para a estrutura P-F com o obtido pela realimentação simples (sem $\mathrm{P})$, mostra-se , como era de se esperar, que em ambos os casos a função de transferência em malha fechada é igual a do modelo de referência. Entretanto, com relação à função de transferência entre $u$ e $v$, mostra-se para estrutura P-F que:

$$
G_{u v}^{P F}=(3 \gamma)^{*}\left(\begin{array}{ll}
e & e \\
e & e
\end{array}\right)
$$

\footnotetext{
${ }^{5}$ Programas para a manipulação da álgebra de dióide usando o pacote computacional Scilab são propostos por Lhommeau e Hardouin (2003).
} 
Para a realimentação simples, como proposto por Cottenceau et al. (1999), obtém-se:

$$
G_{u v}^{F}=\left(\begin{array}{cc}
e \oplus\left(18 \gamma^{6}\right)(3 \gamma)^{*} & \left(18 \gamma^{6}\right)(3 \gamma)^{*} \\
\left(18 \gamma^{6}\right)(3 \gamma)^{*} & e \oplus\left(18 \gamma^{6}\right)(3 \gamma)^{*}
\end{array}\right) .
$$

Observa-se que $G_{u v}^{P F} \succ G_{u v}^{F}$, ou seja o desempenho do sistema controlado para a estrutura P-F, considerando uma política de gestão "just-in-time" é melhor que o desempenho obtido pela realimentação simples. De fato, constata-se esse resultado comparando-se as séries de cada elemento das matrizes em questão. Por exemplo, para o elemento $(1,1)$ da matriz $G_{u v}^{P F}$, têm-se:

$$
\begin{aligned}
G_{u v}^{P F}(1,1)= & 0 \gamma^{0} \oplus 3 \gamma \oplus 6 \gamma^{2} \oplus 9 \gamma^{3} \oplus \ldots \oplus \\
& 18 \gamma^{6} \oplus 21 \gamma^{7} \oplus \ldots
\end{aligned}
$$

Desenvolvendo a série para o mesmo elemento da matriz $G_{u v}^{F}$, obtém-se:

$$
\begin{aligned}
G_{u v}^{F}(1,1)= & 0 \gamma^{0} \oplus 0 \gamma \oplus 0 \gamma^{2} \oplus 0 \gamma^{3} \oplus \ldots \oplus \\
& 18 \gamma^{6} \oplus 21 \gamma^{7} \oplus \ldots
\end{aligned}
$$

Comparando o desenvolvimento das duas séries apresentadas nas equações 27 e 28, observa-se que os seis primeiros termos do elemento $(1,1)$ de $G_{u v}^{P F}$ são maiores do que os do elemento $(1,1)$ de $G_{u v}^{F}$. A partir do sétimo termo em diante as séries se igualam. Essas comparações podem ser feitas para todos os elementos das duas matrizes. A conclusão final é que $G_{u v}^{P F} \succ G_{u v}^{F}$. Isso quer dizer, que a estrutura P-F atende a especificação de demanda retardando mais a entrada de matéria prima do sistema do que a realimentação simples.

\subsection{Controle multivariável generalizado}

Nas estratégias de controle apresentadas nas Seções 4.1, 4.2 e 4.3 anteriores, tem-se controle apenas sobre a variável de entrada $u$ do sistema. Ou seja, o objetivo de controle é atrasar o máximo possível o disparo da transição de entrada de forma a satisfazer uma especificação de saída do sistema. Além disso, se a informação da saída $y$ do sistema é utilizada no cálculo do controle, tem-se a realimentação de saída. Caso contrário, tem-se a pré-compensação, em malha aberta.

Suponha agora que as transições internas do sistema possam ser eventualmente observadas e/ou controladas. Uma transição é observável se se conhece seus instantes de disparo e é controlável se seu disparo puder ser atrasado indefinidamente por uma ação de controle externa. Nesse caso, o objetivo de controle é ligeiramente modificado para atrasar o máximo possível as transições controláveis usando as informações de disparo das transições observáveis de forma a satisfazer uma especificação de saída do sistema (modelo de referência). Neste trabalho, isso é denominado controle multivariável generalizado.

A ação de controle atua sobre as transições internas (estado) do sistema através de uma matriz $R$ e as observa através de uma matriz $Q$. Essas matrizes representam as ligações (acessos) com as transições controláveis e observáveis, respectivamente. Assim, tem-se $x=A x \oplus R x_{c} \oplus B u$, sendo $(A, B, C)$ uma realização do sistema com matrizes de dimensões apropriadas. Os vetores $x, u$ e $y$ são os vetores de estado, de entrada e de saída do sistema, respectivamente. $\mathrm{O}$ vetor $x_{c}=L x_{o}$ é o vetor de saída do controlador $L \mathrm{e}$ $x_{o}=Q x$ o vetor de estado observado. Nesse caso, a seguinte representação no espaço de estado é obtida:

$$
\begin{aligned}
& x=(A \oplus R L Q) x \oplus B u \\
& y=C x
\end{aligned}
$$

Essa configuração é mostrada na Figura 11.

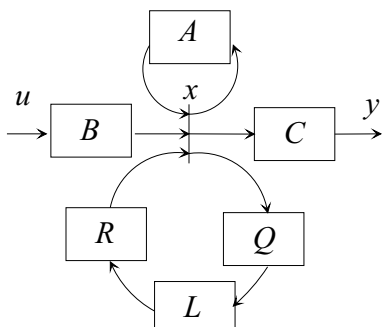

Figura 11: Controle multivariável generalizado.

Note que estrutura em realimentação de saída da Seção 4.2 é obtida do caso geral, bastando fazer $R=B$ e $Q=C$. Conforme a Seção 2.2, a Equação (29a) implícita em $x$ tem como solução mínima $x=(A \oplus R L Q)^{*} B u$. Logo, $y=$ $\left[C(A \oplus R L Q)^{*} B\right] u$ e a matriz de transferência do sistema em malha fechada $G_{c}^{L}$ é, por definição:

$$
G_{c}^{L}=C(A \oplus R L Q)^{*} B
$$

Logo, o problema de controle por modelo de referência utilizando a estrutura da Figura 11 consiste em obter a solução máxima da seguinte inequação em $L$ :

$$
(A \oplus R L Q)^{*} \preceq A_{\text {ref }}
$$

sendo a matriz $A_{r e f}=C \phi G_{r e f} \phi B$ obtida a partir do modelo de referência $G_{r e f}$, aplicando-se o resíduo do produto à esquerda por $C$ e à direita por $B$.

A partir da Equação 31, tem-se $A^{*}\left((R L Q) A^{*}\right)^{*} \preceq A_{\text {ref }}$, pois $(a \oplus b)^{*}=a^{*}\left(b a^{*}\right)^{*}$. Logo, $\left((R L Q) A^{*}\right)^{*} \preceq A^{*} \phi A_{\text {ref }}$, em decorrência do resíduo do produto à esquerda por $A^{*}$. 
Mas, $(R L Q) A^{*} \preceq\left((R L Q) A^{*}\right)^{*}$, pois $a \preceq a^{*}$. Assim, $(R L Q) A^{*} \preceq A^{*} \phi A_{\text {ref }}$ e $R L Q \preceq A^{*} \phi A_{\text {ref }} \phi A^{*}$, em decorrência do resíduo do produto à direita por $A^{*}$. Finalmente, $L \preceq R \phi K \phi Q$, onde $K=A^{*} \phi A_{\text {ref }} \phi A^{*}$, em decorrência dos resíduos dos produtos à esquerda e à direita por $R$ e $L$, respectivamente. Ou seja, $R \phi K \phi Q$ é um limitante superior do conjunto solução da Equação 31. Portanto, se $L_{o p}=R \phi K \phi Q$ é também solução, então $L_{o p}$ é a solução máxima. Maiores detalhes podem ser encontrados em Lüders e Santos-Mendes (2002).

Para ilustrar o uso do resultado acima, considere o seguinte exemplo. Uma empresa de serviço expresso de entrega de encomendas dispõe de um sistema de recepção, inspeção e despacho de pacotes. A recepção é feita na estação $E_{1}$, onde o pacote é descarregado. Esta operação consome uma unidade de tempo para cada pacote e duas ações são disparadas: o cadastramento de recebimento do pacote e o seu encaminhamento para a estação seguinte (inspeção e agendamento da entrega). O cadastramento é feito assim que o pacote é descarregado, independentemente das etapas posteriores.

Quando o pacote chega à estação $E_{2}$ seguinte, outras duas ações se iniciam: a geração de uma etiqueta de agendamento da entrega e a inspeção visual do pacote, como procedimento de segurança. A geração da etiqueta é feita na estação $E_{3}$ que, após a identificação do destino, agenda a entrega. Esta operação consome duas unidades de tempo para cada pacote. $\mathrm{Na}$ estação $E_{4}$ ocorre a inspeção, que consome três unidades de tempo para cada pacote. Finalmente, na estação $E_{5}$, a etiqueta é fixada no pacote, que segue para a seção de despacho. Esse cenário é representado na Figura 12, onde a saída $y_{1}$ representa a ação de cadastramento dos pacotes e a saída $y_{2}$ a liberação dos pacotes para despacho.

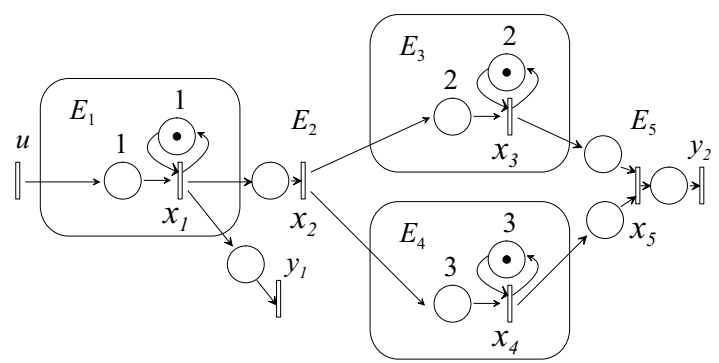

Figura 12: Sistema de entrega de encomendas.

Uma breve análise desse cenário, mostra que haverá um acúmulo de ações de agendamento na estação $E_{3}$ e de pacotes para inspeção na $E_{4}$, pois a taxa de recebimento de pacotes oriundos de $E_{2}$, é maior do que $E_{3}$ e $E_{4}$ podem absorver. Além disso, a geração da etiqueta ocorre mais rapidamente do que a inspeção do pacote, o que provoca um acúmulo de etiquetas na estação $E_{5}$, aguardando a liberação dos pacotes em $E_{4}$.
Uma realização $(A, B, C)$ desse sistema é dada pelas matrizes abaixo:

$$
\begin{aligned}
A & =\left(\begin{array}{ccccc}
1 \gamma & \epsilon & \epsilon & \epsilon & \epsilon \\
e & \epsilon & \epsilon & \epsilon & \epsilon \\
\epsilon & 2 & 2 \gamma & \epsilon & \epsilon \\
\epsilon & 3 & \epsilon & 3 \gamma & \epsilon \\
\epsilon & \epsilon & e & e & \epsilon
\end{array}\right) B=\left(\begin{array}{c}
1 \\
\epsilon \\
\epsilon \\
\epsilon \\
\epsilon
\end{array}\right) \\
C & =\left(\begin{array}{ccccc}
e & \epsilon & \epsilon & \epsilon & \epsilon \\
\epsilon & \epsilon & \epsilon & \epsilon & e
\end{array}\right)
\end{aligned}
$$

Nesse caso, a relação entrada e saída $y=H u$, sendo $H=$ $C A^{*} B$, é dada por:

$$
y=\left(\begin{array}{l}
y_{1} \\
y_{2}
\end{array}\right)=H u=\left(\begin{array}{c}
1(1 \gamma)^{*} \\
4(3 \gamma)^{*}
\end{array}\right) u
$$

Logo, $H=\left[1(1 \gamma)^{*} 4(3 \gamma)^{*}\right]^{\prime}$.

A relação entrada e saída acima mostra que, o cadastramento do primeiro pacote ocorrerá uma unidade de tempo após sua chegada e, posteriormente, a uma taxa de um pacote a cada unidade de tempo. Esse é o tempo necessário para descarregamento dos pacotes. Além disso, o primeiro pacote estará pronto para despacho apenas quatro unidades de tempo após sua chegada e, posteriormente, um pacote poderá ser despachado a cada três unidades de tempo. Basicamente, esse é o tempo necessário para inspeção visual. Isso está representado na Figura 13, pelas respostas impulsivas $y_{1}(k)$ (à esquerda) e $y_{2}(k)$ (à direita).
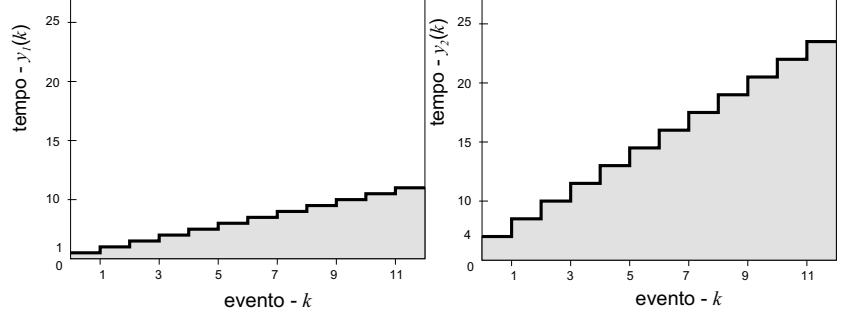

Figura 13: Resposta impulsiva de $H$.

Suponha entretanto que, na saída dos pacotes para a seção de despacho, exista um sistema de transporte cuja taxa de atendimento é menor do que a a capacidade de inspeção e agendamento. Logo, para que não haja formação de estoque no pátio de transporte, deve-se garantir que os pacotes sejam liberados a uma taxa menor. Por exemplo, um pacote a cada cinco unidades de tempo. Porém, o cadastramento de chegada dos pacotes deve manter-se inalterado. Isso significa especificar $G_{r e f}=\left[1(1 \gamma)^{*} 4(5 \gamma)^{*}\right]^{\prime}$. Assim, a trajetória de disparo da saída $y_{1}(k)$ permanece inalterada, enquanto a trajetória de disparo da saída $y_{2}(k)$ é atrasada, conforme mostra a Figura 14 à esquerda e à direita, respectivamente. 


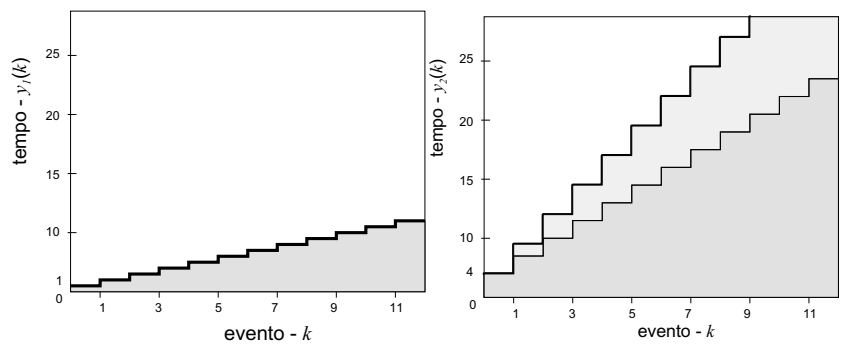

Figura 14: Resposta impulsiva de $G_{r e f}$.

Conforme visto na Seção 4.1, o controlador em précompensação é dado por:

$$
P_{o p}=H \phi G_{r e f}=(1 \gamma)^{*}
$$

Nesse caso, a matriz de transferência do sistema précompensado é $G_{c}^{P}=H$. Ou seja, a pré-compensação atrasa ao máximo a entrada $u$, sem, no entanto, conseguir uma maior aproximação da especificação $G_{r e f}$.

Para o controlador em realimentação, conforme a Seção 4.2, tem-se:

$$
F_{o p}=H \phi G_{r e f} \phi H=\left(1 \gamma(1 \gamma)^{*} \quad \epsilon\right)
$$

Nesse caso, a matriz de transferência do sistema realimentado também permanece inalterada, ou seja, $G_{c}^{F}=H$. Assim, também não se consegue uma maior aproximação da especificação $G_{r e f}$.

Isso ocorre tanto na pré-compensação quanto na realimentação em decorrência da impossibilidade de se atrasar mais a entrada $u$ sem que se altere a taxa de cadastramento dos pacotes na saída $y_{1}$ (que deve permanecer inalterada).

Suponha agora que se tenha acesso a algumas das transições internas do sistema. Por exemplo, a transição $x_{2}$ é controlável e $x_{5}$ é observável. Nesse caso, tem-se $R=\left[\begin{array}{llll}\epsilon & e & \epsilon & \epsilon \in\end{array}\right]^{\prime}$ e $Q=\left[\begin{array}{llll}\epsilon & \epsilon \in & \epsilon\end{array}\right]$.

Calculando-se a matriz $K=A^{*} \phi A_{r e f} \phi A^{*}$ tem-se:

$K=\left(\begin{array}{ccccc}(1 \gamma)^{*} & (1 \gamma)^{*} & \epsilon & \epsilon & \epsilon \\ (5 \gamma)^{*} & (5 \gamma)^{*} & 3 \gamma(5 \gamma)^{*} & 2 \gamma(5 \gamma)^{*} & 2 \gamma(5 \gamma)^{*} \\ 3(5 \gamma)^{*} & 3(5 \gamma)^{*} & 1(5 \gamma)^{*} & (5 \gamma)^{*} & (5 \gamma)^{*} \\ 3(5 \gamma)^{*} & 3(5 \gamma)^{*} & 1(5 \gamma)^{*} & (5 \gamma)^{*} & (5 \gamma)^{*} \\ 3(5 \gamma)^{*} & 3(5 \gamma)^{*} & 1(5 \gamma)^{*} & (5 \gamma)^{*} & (5 \gamma)^{*}\end{array}\right)$

Pode-se mostrar que o controlador $L=R \oint K \phi Q=2 \gamma(5 \gamma)^{*}$ é solução da Equação 31 e, portanto, é o controlador máximo em realimentação de estado com $x_{2}$ controlável e $x_{5}$ observável. Nesse caso, a matriz de transferência do sistema controlado é $G_{c}^{L}=\left[1(1 \gamma)^{*} 4(5 \gamma)^{*}\right]^{\prime}$, ou seja, iguala a especificação $G_{r e f}$. O sistema controlado é mostrado na Figura 15.

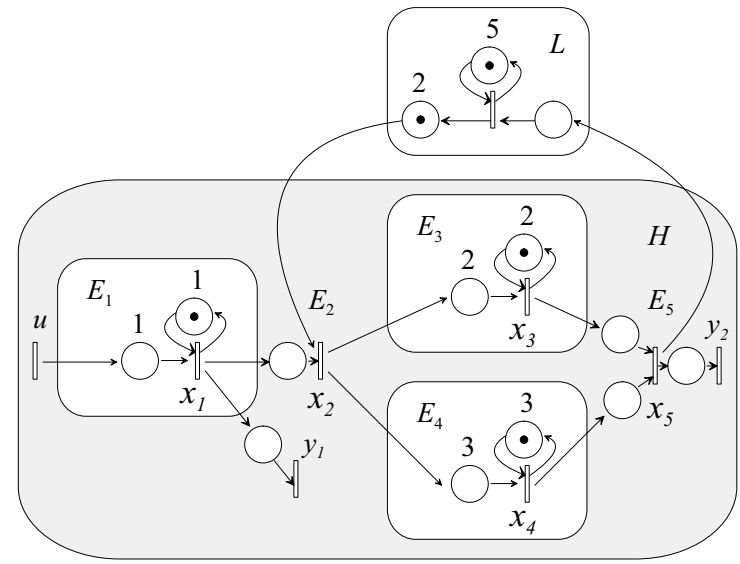

Figura 15: Sistema de entrega controlado.

Em outras palavras, o acesso às transições internas do sistema permitiu que apenas a inspeção e agendamento de pacotes fosse atrasada, satisfazendo a especificação de reduzir a taxa para um pacote a cada 5 unidades de tempo. Nas soluções por pré-compensação e por realimentação não é possível separar o cadastramento (mais rápido) da inspeção e agendamento (mais lento). Nessas configurações, qualquer tentativa de atrasar a chegada dos pacotes, atrasa também o cadastramento.

De maneira geral, o controle multivariável (que observa e atua sobre transições internas do sistema) permite uma ação de controle mais localizada, em pontos de menor interdependência entre as diversas relações de entrada e saída do sistema. Isso traz maior flexibilidade de controle.

Entretanto, o problema de estabilidade permanece, pois sempre ocorrerá acúmulo de fichas no lugar que conecta um subsistema rápido fornecendo fichas para um subsistema mais lento (Commault, 1998). Porém, esta estratégia tem a vantagem de restringir o acúmulo de fichas a determinados lugares do sistema. Para o sistema controlado, note que o subsistema inspeção/agendamento dos pacotes é um componente fortemente conexo do grafo e, portanto, o acúmulo de pacotes ocorrerá apenas no lugar entre as transições $x_{1}$ e $x_{2}$, ou seja, apenas na estação $E_{2}$ (antes do controle, havia acúmulo nas estações $E_{3}, E_{4}$ e $E_{5}$ ).

\section{CONCLUSÕES}

Neste artigo tutorial foram apresentadas e discutidas diversas estratégias de controle por modelo de referência para sistemas a eventos discretos Max-plus lineares. Como principais contribuições para o estudo das alternativas a serem utilizadas como estrutura de controle, destacam-se a estrutura P-F e o controle multivariável generalizado. A estrutura P-F baseia-se na realimentação de saída e tem como prin- 
cipais características o fato de assegurar desempenho ótimo para qualquer modelo de referência e, além disso, favorecer a estabilidade do sistema. O controle multivariável, por sua vez, permite um controle mais localizado, restringindo sua atuação, por exemplo, aos gargalos do sistema. Contudo, há restrições na escolha do modelo de referência, ou seja, o controlador máximo não pode ser obtido para um modelo de referência qualquer, sendo que a metodologia fornece apenas uma condição suficiente para a existência do controlador máximo. Um ponto importante a ser investigado diz respeito à utilização de um pré-compensador em conjunto com a estrutura multivariável proposta, procurando, dessa forma, diminuir as restrições na escolha do modelo. Vale ressaltar que as idéias e os resultados apresentados são válidos para sistemas invariantes e determinísticos. Uma tendência atual e que deve se prolongar para o futuro é a extensão das idéias propostas para sistemas variantes no tempo, assim como para plantas com parâmetros incertos.

\section{AGRADECIMENTOS}

O primeiro autor agradece à PRPq/UFMG pelo suporte financeiro durante a realização deste trabalho.

\section{REFERÊNCIAS}

Antsaklis, A. J. (2000). A brief introduction to the theory and applications of hybrid systems, Proceedings of the IEEE 88(7): 879-887. Special Issue on Hybrid Systems: Theory and Applications.

Baccelli, F., Cohen, G., Olsder, G. e Quadrat, J. (1992). Synchronisation and Linearity: An Algebra for Discrete Event Systems, John Wiley and Sons, New York.

Banks, J., Carson, J. S., Nelson, B. L. e Nicol, D. M. (2000). Discrete-Event System Simulation, 3rd. edn, PrenticeHall.

Blyth, T. e Janowitz, M. (1972). Residuation Theory, Pergamon Press, Oxford.

Boimond, J. e Ferrier, J. (1996). Internal model control and max-algebra : Controller design, IEEE TAC 41(3): 457-461.

Cassandras, C. G. e Lafortune, S. (1999). Introduction to Discrete Event Systems, Kluwer Academic Publishers.

Cohen, G., Gaubert, S. e Quadrat, J.-P. (1999). Max-plus algebra and system theory: where we are and where to go now, Annual Reviews in Control 23: 207-219.

Cohen, G., Moller, P., Quadrat, J. e Viot, M. (1989). Algebraic tools for the performance evaluation of discrete event systems, IEEE Proceedings: Special issue on Discrete Event Systems 77(1): 39-58.

Commault, C. (1998). Feedback stabilization of some event graph models, IEEE Trans. on Automatic Control 43(10): 1419-1423.

Cottenceau, B. (1999). Contribution à la commande de systèmes à événements discrets : synthèse de correcteurs pour les graphes d'événements temporisés dans les dioüdes, Thèse de doctorat, Université d'Angers, Angers - France.

Cottenceau, B., Hardouin, L., Boimond, J. e Ferrier, J. (1999). Synthesis of greatest linear feedback for timed event graphs in dioid, IEEE Trans. on Automatic Control 44(6): 1258-1262.

Cottenceau, B., Hardouin, L., Boimond, J. e Ferrier, J. (2001). Model reference control for timed event graphs in dioid, Automatica 37: 1451-1458.

Cuninghame-Green, R. (1979). Minimax algebra, Lecture notes in Economics and Mathematical Systems, number 166, Springer-Verlag, Berlin.

Hardouin, L. (2004). Sur la Commande linéaire de Systèmes à Evenements Discrets dans l'algèbre (max,+), Habilitation à diriger des recherches, Université d'Angers, Angers - France.

Kleinrock, L. (1975). Queueing Systems - Theory, Vol. I, Wiley-Interscience.

Lahaye, S., Boimond, J. e Hardouin, L. (2004). Linear periodic systems over dioids, Discrete Event Dynamic Systems - Theory and Applications 14(2): 133-152.

Lhommeau, M. e Hardouin, L. (2003). Software tools for manipulating periodic series.

URL: http://www.istia-angers.fr/ hardouin/outils.html

Lhommeau, M., Hardouin, L., Cottenceau, B. e Jaulin, L. (2004). Interval analysis and dioid: application to robust controller design for timed event graphs, Automatica 40: 1923-1930.

Lüders, R. (2001). Controle Multivariável de Sistemas a Eventos Discretos em Dióides, Tese de doutorado, UNICAMP, Campinas,SP, Brasil.

Lüders, R. e Santos-Mendes, R. (2002). Generalized multivariable control of discrete event systems in dioid, 6th International Workshop on Discrete Event Systems (WODES'02), Zaragoza, Spain.

Maia, C. A. (2003). Identificação e Controle de Sistemas a Eventos Discretos na Álgebra (max, +), Tese de doutorado, UNICAMP, Campinas, SP, Brasil. 
Maia, C. A., Hardouin, L., Santos-Mendes, R. e Cottenceau, B. (2003). Optimal closed-loop control of timed event graphs in dioids, IEEE Trans. on Automatic Control 48(12): 2284-2287.

Menguy, E., Boimond, J., Hardouin, L. e Ferrier, J. (2000). A first step towards adaptive control for linear systems in max algebra, Discrete Event Dynamic Systems.Theory and Applications 10: 347-367.

Murata, T. (1989). Petri nets : properties, analysis and applications., Proceedings of the IEEE 77(4): 541-580.

Ostroff, J. (1989). Temporal Logic for Real-Time Systems, John Wiley, New York.

Ramadge, P. e Wonham, W. (1989). The control of discrete event systems, IEEE TAC 77: 81-98.

Wang, J. (1998). Timed Petri Nets, Kluwer Academic Publishers. 